\title{
Suboptimal Adherence and Associated Factors Contributing to Virological Failure on First Line ART at a Private Tertiary Care Centre in South India: Implications for Individualized Adherence Interventions in Resource-limited Settings
}

\section{Laxman G Jessani*, Ram Gopalakrishnan, Suresh D, S Nambi and V Ramasubramanian}

Department of Infectious Diseases, Apollo Hospital, Chennai

*Corresponding Author: Laxman G Jessani, Department of Infectious Diseases, Apollo Hospital, Chennai.
Received: August 26, 2020

Published: September 16, 2020

(C) All rights are reserved by Laxman G Jessani., et al.

\begin{abstract}
Background: Suboptimal adherence to ART can lead to HIV drug resistance. We studied the incidence and factors leading to firstline ART failure and the socio-demographic, behavioral and clinical risk factors associated with non-adherence to ART.

Methods: We carried out a retrospective case-control study of adult HIV-1 positive patients who were on first line ART over a period of 10 years. Adherence and history of treatment interruptions for $>48 \mathrm{~h}$ were noted by self-report. Kaplan Meier survival curves (Figure 2) were used to estimate the median time to first line ART treatment failure. Logistic regression and Cox proportional hazard model were used to predict ART treatment failure.

Results: Among 290 participants, 80 (27.58\%) cumulatively had first-line failure. Patients who had virological failure on first line ART were taken as cases $(n=80)$ and those who were virologically suppressed were taken as controls $(n=210)$. Mean time to failure was $41.16 \pm 11.11$ months. $45 \%$ failed after more than 5 yrs on treatment: only $4 \%$ failed within the first 6 months. Lower baseline CD4 count, WHO stage IV, CDC stage 3 and poor adherence were significantly associated with first line failure. $70 \%$ of patients reported suboptimal adherence while $68 \%$ reported treatment interruptions. Poor adherence was significantly associated with virological failure $(\mathrm{P}<0.001)$ with $\mathrm{OR}=4.22(2.68-6.62)$ and $\mathrm{CD} 4$ count $<200$ and ART duration $>3$ years was associated with poorer adherence.

Conclusion: Our study demonstrates a strong association between suboptimal adherence, virological failure and drug resistance among patients on first-line ART.
\end{abstract}

Keywords: HIV/AIDS; Suboptimal; Adherence; Virological Failure

\section{Introduction}

Antiretroviral therapy (ART) has reduced human immunodeficiency virus (HIV) associated morbidity and mortality by restoring and preserving immunological function [1]. Though there has been a global increase in antiretroviral therapy (ART) roll out, long term treatment carries the potential of drug toxicity and emergence of resistance, resulting in treatment failure. Optimal adherence to ART results in maintaining HIV viral suppression and prevention of development of drug-resistant virus, whereas interruptions in therapy, missed antiretroviral doses and improper dosing can all lead to HIV drug resistance [2].
There is enough evidence that ART adherence rates of $95 \%$ are needed to suppress viral replication and avoid development of HIV drug resistance [2,3]. Studies conducted in resource-limited settings have shown that HIV infected individuals can maintain high levels of adherence to ART [4,5]. Data from Africa [6] and India [7] indicate that ART adherence levels may decline over time. In India, published data on adherence have been limited to a few studies, using cross-sectional designs [8-10], non-standard adherence measures, different adherence cut-offs, whereas there is a paucity of $[8,11]$ quantitative studies examining behavioral correlates with adherence [10]. Besides, studies done in resource-limited settings 
used clinical and immunological monitoring for ART response, rather than virological monitoring [12-14].

We therefore attempted to study incidence and factors causing first-line ART failure, the socio-demographic, behavioral and clinical risk factors associated with non-adherence to ART and examined the longitudinal relationship between ART adherence and the presence of virological failure.

\section{Methods}

We carried out a retrospective case-control study of adult HIV-1 positive patients who had entered clinical care between January 2006 and December 2018 and had remained on follow-up since. Adults aged 18 yrs or more who were on first line ART for at least 6 months were included. Those who had confirmed first line ART virological failure at initial presentation, pregnant patients, those with HIV-2 infection and those with incomplete data were excluded. On follow up, patients who had documented virological failure on first line ART were taken as cases $(n=80)$ and those who were virologically suppressed were taken as controls $(n=210)$.

\section{Study setting and population}

The Infectious Disease Department at a private tertiary care 700 bed hospital in South India, treats approximately 100 new HIV patients annually in the outpatient clinic. The centre provides services such as ART, opportunistic infection prophylaxis, and routine laboratory monitoring tests. Patients are given adherence counseling at each visit. Criteria for ART initiation and switching, as well as ART regimens were as per WHO and US-DHHS guidelines.

\section{Data collection}

Demographic data including gender, age at entry into clinical care, WHO/CDC clinical stage at diagnosis and ART details were recorded from patient medical records using a standardized data extraction form. Serial CD4 count and viral load (VL) done every 6 - 12 months, ART regimen details, opportunistic infections were collected. Adherence and history of treatment interruptions for > $48 \mathrm{~h}$ were noted by self-report.

\section{Cohort description}

The first line ART regimen consisted of 2 nucleoside reverse transcription inhibitors (NRTIs: tenofovir, lamivudine, emtricitabine, didanosine, zidovudine, stavudine and abacavir) and a non-nucleoside reverse transcription inhibitor (NNRTI: either nevirapine or efavirenz). In case of virological failure, second line regimens consisted of a boosted protease inhibitor (PI): either lopinavir/ritonavir or atazanavir/ritonavir with either 2-3 NRTIs or with an integrase-strand transferase inhibitor (raltegravir). Second line ART regimens were usually chosen based on genotypic resistance profiles at time of first line virologic failure.

\section{Laboratory assessment}

Laboratory measurements included a complete blood cell count, CD4 lymphocyte count, and quantitative measurement of HIV load. Viral load count was done with use of Amplicor monitor standard assay, version 1.5 (Roche Molecular Systems), with a minimum detection limit of 40 copies/ml. CD4 lymphocytes were analyzed by flow cytometry.

\section{Definitions}

- $\quad$ Patients on first line therapy were eligible for evaluation for second-line ART if they had been receiving ART for at least 6 months and had clinical, immunological or virological failure. Only those patients that had documented virological failure were analysed as "cases" in the study.

- Adherence greater than $95 \%$ of doses was considered as good or optimal adherence while less than $95 \%$ was taken as suboptimal or poor adherence, as per earlier studies [15].

- Treatment interruption: In addition to estimating pastmonth adherence, participants were asked how many times they had skipped all their medications for $>48 \mathrm{~h}$ since they started on ART.

- Participants who reported either no history of treatment interruptions or $>95 \%$ adherence in the last month were classified as 'optimally adherent'.

- Participants who reported either a history of treatment interruptions or $<95 \%$ adherence in the last month were classified as 'suboptimally adherent'.

- According to WHO and US-DHHS [16] treatment guidelines.

- Clinical failure was the development of an AIDS-defining illness after at least 3 months on ART.

- Immunologic failure was defined as a decrease in CD4 to baseline (or below) after 6 months of therapy, a CD4 count persistently below 100 cells/ul after 6 months of therapy, or a $50 \%$ decline from the ontreatment peak CD4 value [16]

- Virological failure was defined as inability to achieve or maintain suppression of HIV replication to below the detection limit of the assay.

For our study we used virological failure as the determinant of treatment failure. Clinical and immunological failure was also documented. 


\section{Ethical clearance}

Ethical clearance was obtained from the Ethics Committee of the hospital for the study before commencement.

\section{Statistical methods used}

Descriptive statistics were presented as mean \pm standard deviation (SD) for continuous variables and as frequency and percentages for categorical variables. Patient characteristics were described in terms of median with their inter quartile range (IQR) for variables influenced by extreme values. The cumulative incidence of first-line ART failure was ascertained from the proportion of patients with detectable viral load $>40$ copies per ml during follow up. Kaplan Meier survival curves were used to estimate the median time to first line ART treatment failure. Chi-square test and Fisher's exact test was used to compare different ART first line regimens failure rates. Factors associated with treatment failure were ascertained by comparing various variables using the Chi-square and Fisher's exact test for categorical data and Student unpaired $\mathrm{t}$ and Mann Whitney U test for continuous variables. Logistic regression was done to determine the factors that independently predict ART treatment failure. All social demographic and clinical characteristics (variables) were subjected to univariate analysis and factors which were clinically important were included in multivariate analysis (Cox proportional hazard model). Odds ratios of detectable VL (i.e. VL $\geq 40$ copies $/ \mathrm{ml}$ ) and suboptimally adherent patients and other factors were calculated. Chi-square and Fisher's exact test was used to identify significant independent sociodemographic, behavioral, and clinical predictors of ART nonadherence. A $p$ value less than 0.05 was considered statistically significant. All significance levels reported are two-sided. All analyses were done using SPSS (Statistical Package for Social Sciences) software with version 15.0 .

\section{Results}

We studied the records of 700 patients who had been initiated on ART between January 2006 and December 2018. 410 patients were excluded due to key missing data including CD4 counts, viral loads, information on first line ART, pre-existing first line failure. 80 patients had documented virological first line failure and were included in further analysis (Figure 1). 210 patients who were virologically suppressed on first line ART were taken as controls.

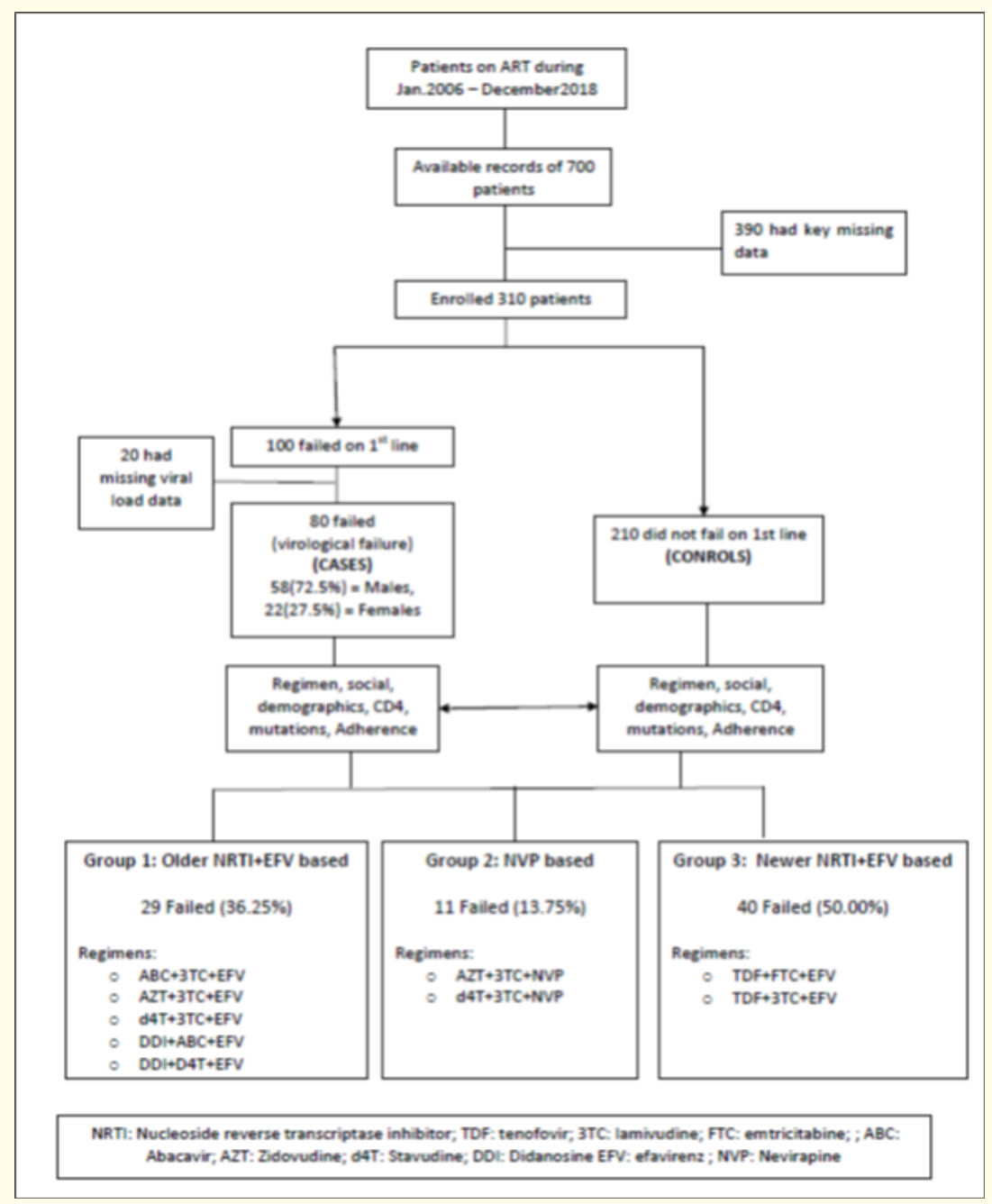

Figure 1 
Suboptimal Adherence and Associated Factors Contributing to Virological Failure on First Line ART at a Private Tertiary Care Centre in South India: Implications for Individualized Adherence Interventions in Resource-limited Settings

\section{Demographic characteristics}

Approximately one third i.e. $98(33.8 \%)$ of the participants were female and the mean age of participants were $41.9 \mathrm{yrs}$. Most participants (55.2\%) were 31 - 45 years of age. More than $90 \%$ of participants were married. Status of partner was not known in $57.9 \%$ cases. $21.7 \%$ were in a HIV-concordant long term relationship (Table 1). At the time of enrollment, the mean HIV-1 viral load was $964,873.15$ copies/ml with a median value of $115,604.5$ copies/ml. The CD4 counts ranged from 77 - 439 cells $/ \mathrm{mm}$, with a median value of 229.5 cells $/ \mathrm{mm}^{3}$ and mean of 282.38 cells $/ \mathrm{mm}^{3}$ with mean CD4 percentage of $17.47 \%$. The study population consisted of individuals predominantly from South India (66.2\%), followed by East India (26.6\%). Nearly 50\% patients were in clinical stage IV WHO HIV staging and CDC stage $3.42 .4 \%$ of participants had tuberculosis, the commonest opportunistic infection, at some point.

\begin{tabular}{|c|c|c|}
\hline \multirow[b]{2}{*}{ Characteristic } & \multicolumn{2}{|c|}{ At ART initiation } \\
\hline & $\begin{array}{l}\text { Frequency } \\
(n=290)\end{array}$ & Percentage (\%) \\
\hline \multicolumn{3}{|l|}{ Age } \\
\hline $18-30$ & 31 & 10.7 \\
\hline $31-45$ & 160 & 55.2 \\
\hline $46-60$ & 81 & 27.9 \\
\hline 61 and above & 18 & 6.2 \\
\hline \multicolumn{3}{|l|}{ Sex } \\
\hline Male & 192 & 66.2 \\
\hline Female & 98 & 33.8 \\
\hline \multicolumn{3}{|l|}{ Marital status } \\
\hline Married & 262 & 90.3 \\
\hline Unmarried & 28 & 9.7 \\
\hline \multicolumn{3}{|l|}{ Residence } \\
\hline South & 192 & 66.2 \\
\hline East & 77 & 26.6 \\
\hline Rest & 21 & 7.2 \\
\hline \multicolumn{3}{|l|}{ Partner HIV status } \\
\hline Concordant & 63 & 21.7 \\
\hline Discordant & 59 & 20.3 \\
\hline Unknown partner status & 168 & 57.9 \\
\hline \multicolumn{3}{|l|}{ CDC Stage } \\
\hline 1 & 91 & 31.4 \\
\hline 2 & 55 & 19.0 \\
\hline 3 & 144 & 49.7 \\
\hline
\end{tabular}

\begin{tabular}{|l|l|l|}
\hline WHO Stage & 72 & \\
II & 51 & 24.8 \\
III & 28 & 17.6 \\
IV & 139 & 9.7 \\
\hline TB Present & 123 & 47.9 \\
\hline $\begin{array}{l}\text { Mean CD4 count absolute } \\
\text { (SD) }\end{array}$ & $282.38(255.7)$ & - \\
\hline $\begin{array}{l}\text { Mean CD4 count percent- } \\
\text { age (SD) }\end{array}$ & 17.47 (9.79) & - \\
\hline Mean Viral load (SD) & 964873.15 & - \\
\hline $\begin{array}{l}\text { ART: Antiretroviral Therapy; TB: Tuberculosis; SD: Standard } \\
\text { Deviation }\end{array}$ \\
\hline
\end{tabular}

Table 1: Baseline clinical and demographic characteristics at ART initiation.

First line ART treatment failure

Among 290 participants, $80(27.58 \%)$ cumulatively had virological first-line ART failure. Patients on newer NRTI+EFV based regimen group had significantly lower first line failure rate (Table $2 \mathrm{a}$ and $2 \mathrm{~b}$ ). The most common first-line regimens among first line failures were $\mathrm{TDF}+\mathrm{FTC}+\mathrm{EFV}$ (34\%), $\mathrm{TDF}+3 \mathrm{TC}+\mathrm{EFV}$ (16\%), $\mathrm{AZT}+3 \mathrm{TC}+\mathrm{EFV}(15 \%)$ and $\mathrm{D} 4 \mathrm{~T}+3 \mathrm{TC}+\mathrm{EFV}(15 \%)$.

\begin{tabular}{|c|c|c|c|}
\hline \multirow{2}{*}{$\begin{array}{l}\text { First line regi- } \\
\text { men }(n=290)\end{array}$} & $\begin{array}{c}\text { Case } \\
(n=80)\end{array}$ & $\begin{array}{c}\text { Control } \\
(n=210)\end{array}$ & \multirow{2}{*}{$\begin{array}{l}\text { Significance } \\
\text { and p value }\end{array}$} \\
\hline & n (\%) & n (\%) & \\
\hline $\begin{array}{l}\text { Group } 1(\mathrm{n}=72) \\
\text { (Older NRTI+ EFV } \\
\text { based) }\end{array}$ & $29(36.3 \%)$ & $43(20.3 \%)$ & \multirow[t]{3}{*}{$\mathrm{P}=0.015^{*}$} \\
\hline $\begin{array}{l}\text { Group } 2(\mathrm{n}=38) \\
(\mathrm{NVP} \text { based })\end{array}$ & $11(13.8 \%)$ & 27 (12.9\%) & \\
\hline $\begin{array}{l}\text { Group } 3(\mathrm{n}=180) \\
(\text { Newer NRTI+EFV } \\
\text { based) }\end{array}$ & $40(50.0 \%)$ & $140(66.7 \%)$ & \\
\hline \multicolumn{4}{|c|}{ Statistical test used: Chi sq. value $=8.4, \mathrm{DF}=2$} \\
\hline
\end{tabular}

Table 2a: First line regimens failure rate comparison among different groups. 
Suboptimal Adherence and Associated Factors Contributing to Virological Failure on First Line ART at a Private Tertiary Care Centre in South India: Implications for Individualized Adherence Interventions in Resource-limited Settings

46

\begin{tabular}{|c|c|c|c|c|}
\hline \multirow{2}{*}{\multicolumn{2}{|c|}{$\begin{array}{l}\text { First line regimen }(n=290) \\
n(\%)\end{array}$}} & \multirow{2}{*}{$\begin{array}{c}\text { Case }(n=80) \\
n(\%)\end{array}$} & \multirow{2}{*}{$\begin{array}{c}\text { Control }(n=210) \\
n(\%)\end{array}$} & \multirow[t]{2}{*}{ Significance and $p$ value } \\
\hline & & & & \\
\hline \multirow{2}{*}{$\begin{array}{l}\text { NRTI comparison (older v/s } \\
\text { newer) }\end{array}$} & Older NRTI based $(\mathrm{n}=110)$ & $40(50.0 \%)$ & $70(33.5 \%)$ & \multirow[t]{2}{*}{$P=0.01^{*}$} \\
\hline & Newer NRTI based $(\mathrm{n}=180)$ & $40(50.0 \%)$ & $140(66.7 \%)$ & \\
\hline \multirow{2}{*}{$\begin{array}{l}\text { NNRTI comparison (EFV v/s } \\
\text { NVP) }\end{array}$} & EFV Based $(n=252)$ & $69(86.3 \%)$ & $183(87.1 \%)$ & \multirow[t]{2}{*}{$\mathrm{P}=0.85$} \\
\hline & NVP based $(n=38)$ & $11(13.8 \%)$ & $27(12.9 \%)$ & \\
\hline \multicolumn{5}{|c|}{ Statistical test used: Fisher Exact Probability Test } \\
\hline
\end{tabular}

Table 2b: First line regimens failure rate comparison among NRTI and NNRTI.

There were no significant differences amongst the three different groups (comprising of older NRTI + EFV based, NVP based, newer NRTI + EFV based) of first line regimen with respect to mean CD4 and viral load at baseline. The mean baseline CD4 counts absolute and percentage at start of ART for those who failed (cases) and remained suppressed throughout (control) were $185.44 \pm$ 207.9 (13.89 $\pm 8.84 \%)$ and $319.31 \pm 262.85$ (18.84 $\pm 9.80 \%)$ respectively (Table 3 ). The median and mean time to failure was 56 and $41.16 \pm 11.11$ months respectively. At the time of switch, the median and mean CD4 cell count was 154 and 204 cells/uL respectively. Amongst those who failed, 36 (45\%) failed after more than 5 yrs, 16 (20\%) each failed between 1 - 3 yrs and 3 - 5 yrs, 9 (11\%) failed between 6 - 12 months and only 3 (4\%) failed within the first 6 months. This signifies that as the duration of ART increases, incidence of first line failure also increases (Figure 2).

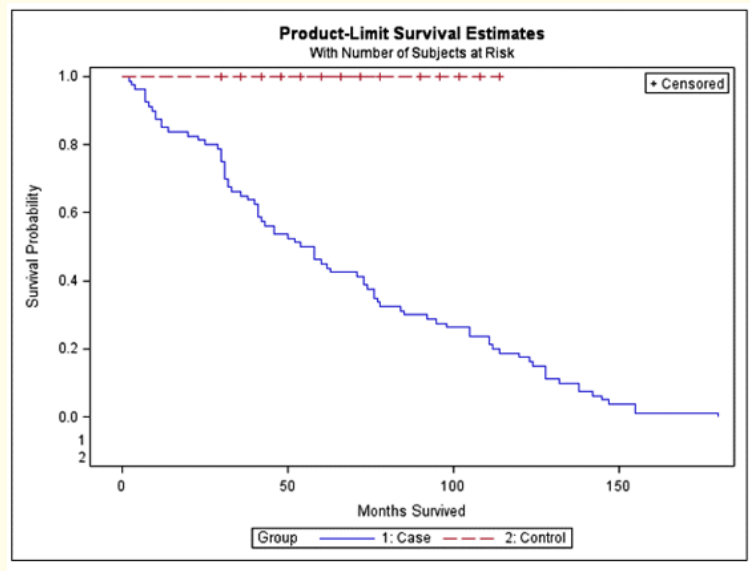

Kaplan Meier Survival Curves (Case=80, Control=210)

Figure 2: Kaplan Meier analysis demonstrating time to first line Art failure.

\begin{tabular}{|c|c|c|c|c|}
\hline $\begin{array}{l}\text { Character- } \\
\text { istic }\end{array}$ & $\begin{array}{l}\text { Cases } \\
\mathbf{n}=\mathbf{8 0}\end{array}$ & $\begin{array}{l}\text { Control } \\
n=210\end{array}$ & $\begin{array}{l}\text { Signif- } \\
\text { icance } \\
\text { and } P \\
\text { value }\end{array}$ & $\begin{array}{l}\text { Odds ratio } \\
95 \% \text { CI and } \\
\text { Signifi- } \\
\text { cance }\end{array}$ \\
\hline $\begin{array}{l}\text { Age (Mean } \\
\pm \text { SD) }\end{array}$ & $\begin{array}{l}41.16 \pm \\
11.11\end{array}$ & $\begin{array}{l}42.42 \pm \\
10.37\end{array}$ & $\begin{array}{l}P= \\
0.30\end{array}$ & - \\
\hline $\begin{array}{l}\text { Age } \\
<45 \\
\geq 45\end{array}$ & $\begin{array}{l}135(64.3 \%) \\
75(35.7 \%) \\
\end{array}$ & $\begin{array}{l}46(57.5 \%) \\
34(42.5 \%)\end{array}$ & - & $\begin{array}{l}1.08 \\
(0.93,1.26) \\
(P>0.05)\end{array}$ \\
\hline $\begin{array}{l}\text { Sex } \\
\text { Male } \\
\text { Female }\end{array}$ & $\begin{array}{l}58(72 \%) \\
22(28 \%)\end{array}$ & $\begin{array}{l}134 \\
(63.8 \%) \\
76(36.2 \%)\end{array}$ & $\begin{array}{l}P= \\
0.17\end{array}$ & $\begin{array}{l}1.35 \\
(0.88,2.06) \\
(P>0.05)\end{array}$ \\
\hline TB present & $35(43.8 \%)$ & $88(61.5 \%)$ & - & $\begin{array}{l}1.30 \\
(1.06,1.60) \\
(P>0.05)\end{array}$ \\
\hline $\begin{array}{l}\text { Adherence } \\
\text { Good } \\
\text { Suboptimal }\end{array}$ & $\begin{array}{l}24(30.0 \%) \\
56(70.0 \%)\end{array}$ & $\begin{array}{l}195 \\
(92.9 \%) \\
15(7.1 \%) \\
\end{array}$ & $\begin{array}{l}\mathrm{P}= \\
0.000^{*}\end{array}$ & $\begin{array}{l}4.22 \\
(2.68,6.62) \\
(P<0.001)^{*}\end{array}$ \\
\hline $\begin{array}{l}\text { Marital } \\
\text { status } \\
\text { Married } \\
\text { Unmarried }\end{array}$ & $\begin{array}{l}73(91.3 \%) \\
7(8.8 \%)\end{array}$ & $\begin{array}{l}189 \\
(90.0 \%) \\
21(10.0 \%)\end{array}$ & $\begin{array}{l}P= \\
0.80\end{array}$ & $\begin{array}{l}1.04 \\
(0.83,1.30) \\
(P>0.05)\end{array}$ \\
\hline $\begin{array}{l}\text { Partner } \\
\text { HIV status } \\
\text { Concor- } \\
\text { dant } \\
\text { Discordant } \\
\text { Unknown }\end{array}$ & $\begin{array}{l}15(18.8 \%) \\
14(17.5 \%) \\
51(63.8 \%)\end{array}$ & $\begin{array}{l}44(21.0 \%) \\
49(23.3 \%) \\
117 \\
(55.7 \%)\end{array}$ & $\mathrm{P}=0.4$ & - \\
\hline
\end{tabular}

Citation: Laxman G Jessani., et al. "Suboptimal Adherence and Associated Factors Contributing to Virological Failure on First Line ART at a Private Tertiary Care Centre in South India: Implications for Individualized Adherence Interventions in Resource-limited Settings". Acta Scientific Microbiology 3.10 (2020): 42-52. 


\begin{tabular}{|c|c|c|c|c|}
\hline $\begin{array}{l}\text { Residence } \\
\text { South } \\
\text { East } \\
\text { Rest }\end{array}$ & $\begin{array}{l}62(77.5 \%) \\
10(12.5 \%) \\
8(10.0 \%)\end{array}$ & $\begin{array}{l}130 \\
(61.9 \%) \\
67(31.9 \%) \\
13(6.2 \%)\end{array}$ & $\begin{array}{l}\mathrm{P}= \\
0.003^{*}\end{array}$ & - \\
\hline $\begin{array}{l}\text { CDC Stage } \\
1 \\
2 \\
3\end{array}$ & $\begin{array}{l}10(12.5 \%) \\
22(27.5 \%) \\
48(60.0 \%)\end{array}$ & $\begin{array}{l}81(38.6 \%) \\
33(15.7 \%) \\
96(45.7 \%)\end{array}$ & $\begin{array}{l}\mathrm{P}< \\
0.001 *\end{array}$ & - \\
\hline $\begin{array}{l}\text { WHO } \\
\text { Stage } \\
\text { I } \\
\text { II } \\
\text { III } \\
\text { IV }\end{array}$ & $\begin{array}{l}0(0.0 \%) \\
19(23.8 \%) \\
13(16.3 \%) \\
48(60.0 \%)\end{array}$ & $\begin{array}{l}72(34.3 \%) \\
32(15.2 \%) \\
15(7.1 \%) \\
91(43.3 \%)\end{array}$ & $\begin{array}{l}\mathrm{P}< \\
0.001 *\end{array}$ & - \\
\hline $\begin{array}{l}\text { Initial CD4 } \\
\text { absolute } \\
\text { Mean } \pm \text { SD } \\
\text { Median } \\
(\text { IQR) }\end{array}$ & $\begin{array}{l}185.44 \pm \\
207.9 \\
116.5 \\
\\
(50.5- \\
219.5)\end{array}$ & $\begin{array}{l}319.31 \pm \\
262.85 \\
305.0 \\
\\
(79.0- \\
477.0) \\
\end{array}$ & $\begin{array}{l}\mathrm{P}< \\
0.001^{*}\end{array}$ & - \\
\hline $\begin{array}{l}\text { Initial CD4 } \\
\text { percentage } \\
\text { Mean } \pm \text { SD } \\
\text { Median } \\
(\text { IQR) }\end{array}$ & $\begin{array}{l}13.89 \pm 8.84 \\
14.0 \\
(8.64 \\
-15.30)\end{array}$ & $\begin{array}{l}18.84 \pm \\
9.80 \\
18.0 \\
\\
(11.0- \\
25.25) \\
\end{array}$ & $\begin{array}{l}\mathrm{P}< \\
0.001^{*}\end{array}$ & - \\
\hline $\begin{array}{l}\text { Initial viral } \\
\text { load } \\
\text { Mean } \pm \text { SD } \\
\text { Median } \\
\text { (IQR) }\end{array}$ & $\begin{array}{l}531196.8 \pm \\
1382382.9 \\
\\
235000 \\
(67510- \\
1531197)\end{array}$ & $\begin{array}{l}1130083 \pm \\
4217207.1 \\
84000 \\
(1600- \\
860000)\end{array}$ & $\begin{array}{l}P= \\
0.20\end{array}$ & - \\
\hline $\begin{array}{l}\text { Months on } \\
\text { first line } \\
\text { ART } \\
\text { Mean } \pm \text { SD } \\
\text { Median } \\
\text { (IQR) }\end{array}$ & $\begin{array}{l}41.16 \pm \\
11.11 \\
56.0 \\
(30.25- \\
105.0)\end{array}$ & $\begin{array}{l}42.42 \pm \\
10.37 \\
54.0 \\
\\
(36.0- \\
90.0)\end{array}$ & $\begin{array}{l}\mathrm{P}= \\
0.40\end{array}$ & - \\
\hline \multicolumn{5}{|c|}{$\begin{array}{l}\text { Statistical test used: Chi square; Fisher exact test; student un- } \\
\text { paired t-test and Mann Whitney u test }\end{array}$} \\
\hline
\end{tabular}

Table 3: Baseline clinical and demographic characteristic of the study participants.
Factors associated with first line treatment failure and adherence rate

The majority of the patients who failed first-line ART $(n=80)$ were male (72\%) as shown in table 3. Lower CD4 count (absolute and percentage) at baseline, WHO stage IV, CDC stage 3 and poor adherence was significantly associated with first line failure. Median higher viral load at baseline was seen with first line failure but it was not statistically significant. Patients residing in South had statistically significant higher chances of first line failure.

In an attempt to predict virological failure with the help of binary logistic regression model using predictor variables: age, sex, duration of first line ART, association with TB, CDC and WHO stage, adherence, baseline CD4 count and baseline viral load, it was found that none of the variables could independently predict virological failure. Baseline CD4 turned out to be marginally not significant ( $\mathrm{P}$ $=0.052$ ). The model could predict $97.2 \%$ cases correctly.

Among overall 290 patients on first line ART, 27.9\% ( $n=81$ ) patients had either suboptimal adherence or missed doses, whereas among 80 failed cases $70 \%(n=56)$ reported suboptimal adherence i.e. taking $<95 \%$ of their medications in the past month, whereas $68 \%(n=54)$ reported a history of at least one treatment interruption $>48 \mathrm{~h}$ (missed doses). $50 \%$ of those who failed first line ART had irregular follow up.

Overall, patients on newer NRTI+EFV based regimen group had significantly better adherence as compared to other regimens. Even among those who failed first line ART, better adherence was noted in patients on newer NRTI+EFV based regimen group and thus this group also had lower first line failure rate as compared with other regimens (Table 4 and 5).

\begin{tabular}{|c|c|c|c|}
\hline \multirow[t]{2}{*}{$\begin{array}{l}\text { First line regi- } \\
\text { men }(n=290)\end{array}$} & $\begin{array}{c}\text { Good adher- } \\
\text { ence } \\
(n=219)\end{array}$ & $\begin{array}{c}\text { Poor adher- } \\
\text { ence } \\
(n=71)\end{array}$ & \multirow[t]{2}{*}{$\begin{array}{l}\text { Signifi- } \\
\text { cance and } \\
\text { p value }\end{array}$} \\
\hline & n (\%) & n (\%) & \\
\hline Group $1(n=72)$ & & & \\
\hline $\begin{array}{l}\text { (Older NRTI+ } \\
\text { EFV based) }\end{array}$ & $40(18.3 \%)$ & $32(45.1 \%)$ & $\mathrm{P}=0.015^{*}$ \\
\hline $\begin{array}{l}\text { Group } 2(\mathrm{n}=38) \\
(\mathrm{NVP} \text { based })\end{array}$ & $28(12.8 \%)$ & $10(14.1 \%)$ & \\
\hline $\begin{array}{l}\text { Group } 3(n= \\
180)\end{array}$ & & & \\
\hline $\begin{array}{l}\text { (Newer } \\
\text { NRTI+EFV } \\
\text { based) }\end{array}$ & $151(68.9 \%)$ & $29(40.8 \%)$ & \\
\hline \multicolumn{4}{|c|}{ Statistical test used: Chi sq. value $=22.4, \mathrm{DF}=2$} \\
\hline *Significant & & & \\
\hline
\end{tabular}

Table 4: Proportion of patients who had poor adherence among different groups on first line ART.

Citation: Laxman G Jessani., et al. "Suboptimal Adherence and Associated Factors Contributing to Virological Failure on First Line ART at a Private Tertiary Care Centre in South India: Implications for Individualized Adherence Interventions in Resource-limited Settings". Acta Scientific Microbiology 3.10 (2020): 42-52. 
Suboptimal Adherence and Associated Factors Contributing to Virological Failure on First Line ART at a Private Tertiary Care Centre in South India: Implications for Individualized Adherence Interventions in Resource-limited Settings

\begin{tabular}{|l|c|c|c|}
\hline \multirow{2}{*}{ Cases (n = 80) } & Good adherence (n = 24) & Poor adherence (n = 56) & Significance and p value \\
\cline { 2 - 3 } $\begin{array}{l}\text { Group 1 (n = 29) } \\
\text { (Older NRTI+ EFV based) }\end{array}$ & $\mathbf{n}(\mathbf{\%})$ & $25(44.6 \%)$ & \\
\hline $\begin{array}{l}\text { Group 2 (n = 11) } \\
\text { (NVP based) }\end{array}$ & $6(25.0 \%)$ & $5(8.9 \%)$ & \\
\hline $\begin{array}{l}\text { Group 3 (n = 40) } \\
\text { (Newer NRTI+EFV based) }\end{array}$ & $14(58.3 \%)$ & $26(46.4 \%)$ & \\
\hline $\begin{array}{l}\text { Statistical test used: Chi sq. value=7.3, DF=2 } \\
\text { *Significant }\end{array}$ & & \\
\hline
\end{tabular}

Table 5: Proportion of patients who had poor adherence among different groups who failed first line ART (Cases).

Relationship between adherence and virological failure

Out of 80 patients who had virological failure, clinical and immunological failure was seen in $33 \%$ and $80 \%$ respectively. As seen in table 3, poor adherence were significantly associated with virological failure with $70 \%$ of suboptimally or poor adherent patients compared with only $30 \%$ of optimally adherent patients showing virological failure $(\mathrm{P}<0.001)$ with Odd's ratio $=4.22(2.68,6.62)$.
Factors associated with poor adherence

None of the socio-demographic, behavioral or clinical risk factor was significantly associated with poor adherence among patients who failed first line ART (Table 6). CD4 count $<200$ and duration on ART for more than 3 years was associated with poorer adherence but was not statistically significant (Table 7). Distribution of causes leading to poor adherence is seen in table 7, multifactorial being the commonest.

\begin{tabular}{|c|c|c|c|}
\hline \multirow{2}{*}{ Factors } & \multicolumn{2}{|c|}{ Proportion of Adherence in Cases $(n=80)$} & \multirow{2}{*}{ Significance and $p$ value } \\
\hline & Good adherence $(n=24)$ & Poor adherence $(n=56)$ & \\
\hline \multicolumn{4}{|l|}{ Age (yrs) } \\
\hline$<30$ & $2(8.3 \%)$ & $6(10.7 \%)$ & $P=0.9$ \\
\hline $30-45$ & $13(54.2 \%)$ & $25(44.6 \%)$ & \\
\hline $45-60$ & $8(33.3 \%)$ & $22(39.3 \%)$ & \\
\hline$>60$ & $1(4.2 \%)$ & $3(5.4 \%)$ & \\
\hline \multicolumn{4}{|l|}{ Sex } \\
\hline Female & $9(36.66 \%)$ & $13(22.85 \%)$ & $P=0.27$ \\
\hline Male & $15(63.34 \%)$ & $43(77.15 \%$ & \\
\hline TB present & $12(50 \%)$ & $23(41.1 \%)$ & $P=0.27$ \\
\hline \multicolumn{4}{|l|}{ CD4 count at time of failure } \\
\hline$>500$ & $1(4.2 \%)$ & $6(10.7 \%)$ & \\
\hline $350-500$ & $0(0.0 \%)$ & $2(3.6 \%)$ & $P=0.6$ \\
\hline $350-200$ & $5(20.8 \%)$ & $10(17.9 \%)$ & \\
\hline$<200$ & $18(75.0 \%)$ & $38(67.9 \%)$ & \\
\hline \multicolumn{4}{|l|}{ Time on first line ART } \\
\hline Within first 6 months & $0(0.0 \%)$ & $3(5.4 \%)$ & \\
\hline $6-12$ months & $4(16.7 \%)$ & $5(8.9 \%)$ & $\mathrm{P}=0.09$ \\
\hline $1-3$ yrs & $8(33.3 \%)$ & $8(14.3 \%)$ & \\
\hline More than 3 yrs & $12(50.0 \%)$ & $40(71.4 \%)$ & \\
\hline
\end{tabular}

Table 6: Association between ART poor adherence and selected socio-demographic, behavioral and clinical risk factors among first line failure (Cases). 
Suboptimal Adherence and Associated Factors Contributing to Virological Failure on First Line ART at a Private Tertiary Care Centre in South India: Implications for Individualized Adherence Interventions in Resource-limited Settings

\begin{tabular}{|c|c|c|}
\hline Causes & n (\%) & Significance and p value \\
\hline Alcohol & $4(5 \%)$ & \multirow[t]{8}{*}{$\mathrm{P}<0.001^{*}$} \\
\hline Financial & $10(12.5 \%)$ & \\
\hline Pill burden & $8(10 \%)$ & \\
\hline Psychological & $8(10 \%)$ & \\
\hline Side effects & $10(12.5 \%)$ & \\
\hline Travel distance & $10(12.5 \%)$ & \\
\hline Multifactorial & $31(37.5 \%)$ & \\
\hline Total & $81(100 \%)$ & \\
\hline \multicolumn{3}{|c|}{ Statistical test used: Chi-square test } \\
\hline *Significant & & \\
\hline
\end{tabular}

Table 7: Causes for poor adherence or treatment interruptions

$$
(n=290) \text {. }
$$

\section{Discussion}

In our study, the cumulative incidence of first line ART failure was $27.58 \%$, with a median time to first line ART virological failure of 56 months of follow up, comparable to other studies in low income countries with a high prevalence of HIV $[12,17,18]$. A study in rural South Africa reported virological failure rates of 38\%, with a median duration of NNRTI based regimen of 31 months of treatment [12]. Similarly, a comparable rate of 33\% was reported in a Thai study within 96 weeks of follow up [18]. In our study, patients were less likely to fail on a newer NRTI + EFV regimen (i.e. either TDF/FTC/3TC as NRTI backbone combined with EFV as NNRTI) compared to older regimens including AZT, D4T, DDI or NVP based regimen. This could be due to better adherence as pill burden decreases.

We analyzed factors causing first line ART failure. Treatment failure rate was not statistically significant between males and females. A cohort in a similar setting in Uganda reported that males were more likely to fail on ART compared to females [17]. Relation between variables and treatment failure showed that poor adherence, residential status, low baseline CD4 count, low WHO stage (III/IV), low CDC stage (stage 3 ) had statistically significant association with higher incidence of treatment failure comparable to other studies [19]. Other variables that did not show any statistically significant association were higher median baseline viral load, age, marital status, partner HIV status. Presence of TB didn't increase the chance of first line failure in our study. Low baseline CD4 count and baseline WHO stage III/IV, CDC stage 3, are indicators of poor immunological and clinical status of the patients respectively. High viral load at baseline as a risk factor for virological failure was recently observed in the results of TREAT Asia HIV observational database [20] although in our study it was not evident statistically probably due to a smaller number of study participants. Treatment failure occurs with longer periods of ART exposure due to accumulation of minor viral populations over time [21]. Other studies also noted similar finding $[19,21]$. In our study, patients who failed had slightly longer duration of ART but were not statistically significant probably due to smaller number of patients in cases group.

We also looked at the relationship between poor adherence and first line ART failure. One of the factors that were independently associated with treatment failure was poor adherence to ART. The critical role of adherence in the treatment of HIV has been demonstrated in many studies [35]. Antiretroviral therapy (ART) adherence is a strong predictor of biological (virological and immunological) and eventual clinical outcome [15]. In our study, patients with poor adherence were four times more likely to fail as compared with good adherence. Using virological monitoring we clearly demonstrated that $70 \%$ of suboptimally adherent patients versus $30 \%$ of optimally adherent patients showed virological failure suggesting that poor adherence was significantly associated with virological failure. These results replicate and extend other study findings $[7,22]$ suggesting that poor adherence leads to virological failure which thereby also suggests a significant association between adherence and antiretroviral drug resistance.

Studies conducted in resource-limited settings have shown that HIV infected individuals can maintain high levels of adherence to ART [4-6]. One study in Malawi concluded that, based on selfreport, $94 \%$ of the participants reported $100 \%$ adherence in the past 4 days. Moreover $<80 \%$ adherence was significantly associated with a detectable viral load (VL) [23]. This confirms that adherence of $<80 \%$ may be detrimental for long-term treatment success even amongst individuals on fixed-dose combination ART. In our study, among failed cases 70\% reported taking < $95 \%$ of their medications in the past month, whilst $68 \%$ reported a history of at least one treatment interruption $>48 \mathrm{~h}$ (missed doses). Similar to one study [7] we found $>48 \mathrm{~h}$ treatment interruptions remain one of the common form of non-adherence in this setting, suggesting that adherence interventions in India should focus on facilitating uninterrupted adherence rather than only targeting occasional missed doses. We also found that irregular follow up results in

Citation: Laxman G Jessani., et al. "Suboptimal Adherence and Associated Factors Contributing to Virological Failure on First Line ART at a Private Tertiary Care Centre in South India: Implications for Individualized Adherence Interventions in Resource-limited Settings". Acta Scientific Microbiology 3.10 (2020): 42-52. 
Suboptimal Adherence and Associated Factors Contributing to Virological Failure on First Line ART at a Private Tertiary Care Centre in South India: Implications for Individualized Adherence Interventions in Resource-limited Settings

poor adherence and contribute to failure. A study from western India conducted at private health clinics found that among patients paying for treatment out-of pocket, three fourths of patients had 95\% adherence or higher [10]. Our study found that only about $30 \%$ of the participants who had first line failure had greater than 95\% adherence, which is lower than some studies conducted in resource-limited settings [4] However, our findings are consistent with other studies both in India $[7,9]$ and in other resource-limited settings $[4,7,9,24]$.

We also analyzed factors associated with non-adherence. Newer NRTI+ EFV regimen i.e. TDF/FTC/3TC backbone combined with EFV was significantly associated with better adherence and thereby subsequently fewer failure as compared to older NRTI or NVP group. In the current follow-up study, we found that although not statistically significant, but patients with CD4 cell counts $<200$ and on longer ART treatment were more likely to be non-adherent, suggesting possible treatment exhaustion and thereby highlighting the need for sustained efforts to maintain high levels of adherence over time well after the initial immune-restorative effects of treatment. In accordance with earlier studies in resource-limited studies demographic characteristics, such as age, gender, residential status, presence of TB (which increases pill burden) did not predict treatment adherence. Cost of treatment has frequently been cited as a major barrier to adequate adherence in resourcelimited settings [4], including in our patient population [25]. Alcohol use can diminish qualify of life, decrease adherence to medical regimens, and is a concern among HIV-infected individuals [26]. Data have suggested the deleterious consequences of alcohol use on markers of immunological functioning and viral suppression, which could be moderated by nonadherence [27]. The findings of this study suggest that proactive screening and referral for counseling about alcohol use should be a component of HIV care in this regional setting [28].

Earlier studies from different regional settings have demonstrated that psychological distress is a barrier to optimal adherence [29].

In our study, some participants who reported optimal adherence and no treatment interruptions were still failing therapy. This can be partially explained due to lack of comprehensive records of adherence since initiation of therapy and they were probably silently developing resistance mutations. Also, as shown in other studies [7], adherence levels frequently decline over time, suggesting that patients may require assistance to maintain optimal levels over the long-term. Although it is possible that these patients may have been infected with drug-resistant virus, this is unlikely to account for all mutations given the low rate of transmitted resistance in India, which has ranged from $0 \%$ to $14 \%$ in previous studies [30]. Quantitative studies examining behavioral correlates of adherence remain limited in India [10]. Financial issues, difficulty in accessing medicines if residing in rural areas and social stigma are other potential contributors to nonadherence.

Thus our study findings demonstrate a strong association between suboptimal adherence, virological failure and drug resistance among patients on first-line ART. We need to develop a better understanding of the structural, interpersonal, culture-specific, regional and individual barriers to long-term uninterrupted adherence. Given the lack of widely available second line ART in this setting, this may be the best option for minimizing further drug resistance and maintaining the efficacy of first-line drugs in India and other resource-limited settings.

Being a retrospective case control study, we could not control for some of the possible confounders. Secondly baseline resistance testing was not done, so the exact impact of primary drug resistance prior to ART initiation and the accumulation of RAMs is unknown. Since the current study was cross sectional in design it may not reflect the dynamic nature of adherence, which can vary over time. We assessed adherence based on patient self-report [24] which may not perfectly reflect actual adherence levels.

\section{Conclusion and Recommendations}

Adherence to ART should be emphasized at all levels of care in ART service providers to prevent the development of treatment failure and ensure long-term treatment efficacy. Our findings emphasize the essential role for viral load monitoring in detecting early failure and the need for its widespread rollout across public health programs. In this study, we identified several modifiable behavioral risk factors of ART adherence such as advanced stage disease, low CD4 count and older NRTI regimens, suggesting that health care providers could play a pivotal role in integrating individualized adherence interventions to improve treatment outcomes. 


\section{Bibliography}

1. Patel K., et al. "Long-term effectiveness of highly active antiretroviral therapy on the survival of children and adolescents with HIV infection: a 10-year follow-up study". Clinical Infectious Diseases 46.4 (2008): 507-515.

2. Lucas GM. "Antiretroviral adherence, drug resistance, viral fitness and HIV disease progression: a tangled web is woven". Journal of Antimicrobial Chemotherapy 55.4 (2005): 413-416.

3. Arnsten JH., et al. "Antiretroviral therapy adherence and viral suppression in HIV-infected drug users: comparison of self-report and electronic monitoring". Clinical Infectious Diseases 33.8 (2001): 1417-1423.

4. Mills EJ., et al. "Adherence to antiretroviral therapy in subSaharan Africa and North America: a meta-analysis". JAMA 296.6 (2006): 679-690.

5. Diabate S., et al. "Determinants of adherence to highly active antiretroviral therapy among HIV-1-infected patients in Cote d'Ivoire". AIDS 21.13 (2007): 1799-1803.

6. Gill CJ., et al. "No room for complacency about adherence to antiretroviral therapy in sub-Saharan Africa". AIDS 19.12 (2005): 1243-1249.

7. Ekstrand ML., et al. "Developing useful highly active antiretroviral therapy adherence measures for India: the Prerana study". Journal of Acquired Immune Deficiency Syndromes 53.3 (2010): 415-416.

8. Cauldbeck MB., et al. "Adherence to anti-retroviral therapy among HIV patients in Bangalore, India". AIDS Research Therapy 6 (2009): 7.

9. Sarna A., et al. "Adherence to antiretroviral therapy and its determinants amongst HIV patients in India". Indian Journal of Medical Research 127.1 (2008): 28-36.

10. Shah B., et al. "Adherence to antiretroviral therapy and virologic suppression among HIV-infected persons receiving care in private clinics in Mumbai, India". Clinical Infectious Diseases 44.9 (2007): 1235-1244.

11. Sharma M., et al. "Access, adherence, quality and impact of ARV provision to current and ex-injecting drug users in Manipur (India): an initial assessment". International Journal of Drug Policy 18.4 (2007): 319-325.

12. Barth RE., et al. "Long-term outcome of children receiving antiretroviral treatment in rural South Africa: substantial virologic failure on first-line treatment". Pediatric Infectious Disease Journal 30.1 (2011): 52-56.
13. Reynolds SJ., et al. "Failure of immunologic criteria to appropriately identify antiretroviral treatment failure in Uganda". AIDS 23.6 (2009): 697-700.

14. Emmett SD., et al. "Predicting virologic failure among HIV1-infected children receiving antiretroviral therapy in Tanzania: a cross-sectional study". Journal of Acquired Immune Deficiency Syndromes 54.4 (2010): 368-375.

15. Bangsberg DR., et al. "Non-adherence to highly active antiretroviral therapy predicts progression to AIDS". AIDS 15.9 (2001): 1181-1183.

16. WHO: Antiretroviral therapy for HIV infection in adults and adolescents: recommendation for a public health approach (2015).

17. Jittamala P., et al. "Predictors of virologic failure and genotypic resistance mutation patterns in thai children receiving non-nucleoside reverse transcriptase inhibitor-based antiretroviral therapy". Pediatric Infectious Disease Journal 28.9 (2009): 826-830.

18. Kamya MR., et al. "Predictors of long-term viral failure among ugandan children and adults treated with antiretroviral therapy". Journal of Acquired Immune Deficiency Syndromes 46.2 (2007): 187-193.

19. Ekstrand ML., et al. "Suboptimal adherence associated with virological failure and resistance mutations to first-line highly active antiretroviral therapy (HAART) in Bangalore, India". International Health 3.1 (2011): 27-34.

20. Boettiger DC., et al. "Efficacy of second-line antiretroviral therapy among people living with HIV/AIDS in Asia: results from the TREAT Asia HIV observational database". Journal of Acquired Immune Deficiency Syndromes 68.2 (2015): 186195.

21. Kumarasamy N., et al. "High frequency of clinically significant mutations after first-line generic highly active antiretroviral therapy failure: implications for second-line options in resource-limited settings". Clinical Infectious Diseases 49.2 (2009): 306-309.

22. Pujades-Rodriguez M., et al. "Treatment failure and mortality factors in patients receiving second-line HIV therapy in resource-limited countries". JAMA 304.3 (2010): 303-312.

23. Ferradini L., et al. "Scaling up of highly active antiretroviral therapy in a rural district of Malawi: an effectiveness assessment". Lancet 367.9519 (2006): 1335-1342. 
24. Oyugi JH., et al. "Multiple validated measures of adherence indicate high levels of adherence to generic HIV antiretroviral therapy in a resource-limited setting". Journal of Acquired Immune Deficiency Syndromes 36.5 (2004): 11001102.

25. Kumarasamy N., et al. "Barriers and facilitators to antiretroviral medication adherence among patients with HIV in Chennai, India: a qualitative study". AIDS Patient Care STDS 19.8 (2005): 526-537.

26. Hendershot CS., et al. "Alcohol use and antiretroviral adherence: review and meta-analysis". Journal of Acquired Immune Deficiency Syndromes 52.2 (2009): 180-202.

27. Samet JH., et al. "Alcohol consumption and HIV disease progression". Journal of Acquired Immune Deficiency Syndromes 46.2 (2007): 194-199.

28. Strauss SM., et al. "HIV care providers' implementation of routine alcohol reduction support for their patients". AIDS Patient Care STDS 23.3 (2009): 211-218.

29. Mellins CA., et al. "Adherence to antiretroviral medications and medical care in HIV-infected adults diagnosed with mental and substance abuse disorders". AIDS Care 21.2 (2009): 168-177.

30. Balakrishnan P., et al. "HIV type 1 genotypic variation in an antiretroviral treatment-naive population in southern India". AIDS Research and Human Retroviruses 21.4 (2005): 301-305.

\section{Assets from publication with us}

- Prompt Acknowledgement after receiving the article

- Thorough Double blinded peer review

- Rapid Publication

- Issue of Publication Certificate

- High visibility of your Published work

Website: https://www.actascientific.com/

Submit Article: https://www.actascientific.com/submission.php Email us: editor@actascientific.com

Contact us: +919182824667 